\title{
Le secteur des productions animales en Afrique subsaharienne des Indépendances à 2020. II. Approche des échanges par zones sous-régionales
}

\author{
G. Tacher ${ }^{1}$ L. Letenneur ${ }^{1,2}$
}

\begin{abstract}
Mots-clés
Production animale - Consommation alimentaire - Importation - Exportation Zonage - Protéine animale - Offre Afrique au sud du Sahara.
\end{abstract}

\begin{abstract}
Résumé
Pour préciser la grande diversité des échanges et des consommations à l'intérieur de I'Afrique subsaharienne, un zonage en sept sous-régions (O uest, Centre-O uest, Centre-N ord, Est, Centre, Sud, Madagascar) a été réalisé à partir de la demande en produits animaux, de l'homogénéité et de la complémentarité des échanges, et en considérant les unions politiques. Importations et exportations portent en majorité sur les viandes avec une grande diversité selon les zones: solde excédentaire pour la zone orientale, solde déficitaire en Afrique centrale. Toutes les sous-régions identifiées sont déficitaires en produits laitiers avec un déficit global de 428 millions de dollars américains en 1994. Les cuirs et les peaux représentent 15 p. 100 des exportations, et 67 p. 100 de celles-ci sont réalisées par l'Afrique australe. Elles représentent un solde positif et compensent les importations nettes de volailles. Les consommations examinées par sous-régions montrent plusieurs types de consommation et une grande diversité dans les quantités de protéines animales consommées par jour et par habitant allant de 4,2 $\mathrm{g}$ en zone centrale à $13,8 \mathrm{~g}$ dans la zone de I'Igad (Intergovernmental Authority on Drought). Les évolutions des consommations montrent une forte augmentation de la consommation des viandes blanches dans les régimes alimentaires dans la plupart des zones. L'offre montre une grande diversité entre les sept zones tant dans la composition et les effectifs des espèces animales et des produits que dans les importations. Les deux grandes sous-régions importatrices sont l'Afrique centrale et l'A frique australe.
\end{abstract}

\section{INTRO DUCTION}

Du bilan global des exportations, des importations, de la consommation et de l'offre en produits animaux de l'Afrique subsaharienne (Ass) exposée dans la partie I (14), il se dégage une participation limitée de cette grande région dans les échanges mondiaux et une satisfaction insuffisante des consommateurs en protéines d'origine animale. Cette réalité ne montre pas la grande diversité qui existe dans les effectifs des cheptels et les échanges selon les pays et les sous-régions. Pour affiner une approche par zones d'échanges et mieux préciser les voies de développement des productions animales, un zonage de l'Ass a été établi sur les bases suivantes :

- la demande, considérée comme déterminante pour le futur développement des filières. C'est ainsi qu'en général un pays est le pôle qui drainera la production disponible pour les exportations intrazones (Côte d'Ivoire, Nigeria, Afrique du Sud) $(2,3,6,9,13)$;

1. Cirad-emvt, TA 30/B, Campus international de Baillarguet, 34398 Montpellier Cedex 5, France

2. E-mail : leon.letenneur@cirad.fr
- l'homogénéité et la complémentarité des échanges de produits animaux $(8,10,11,12)$. La priorité a été donnée aux échanges intrazones, les échanges inter-zones étant le plus souvent marginaux ;

- les unions politiques. Elles donnent une homogénéité commerciale à des sous-régions (Igad $*, \operatorname{Sadc} * *)$.

Sur ces bases, et en prenant les mêmes méthodes $(13,14)$ et références statistiques $(4,5,9)$ que pour la première partie, l'Afrique subsaharienne a été divisée en sept zones. Dans cette classification, la délimitation entre deux zones est quelquefois ambiguë car un courant commercial peut exister avec une autre zone (un pays pouvant exporter sur deux zones à la fois), mais nous avons préféré un certain arbitraire en définissant les zones à partir de pays (unité de base pour des raisons politiques et statistiques) plutôt que de couper certains pays en deux où aucune base statistique n'aurait pu venir étayer une description chiffrée des productions. De plus, les mesures à prendre ne peuvent, dans le cadre de cette étude, qu'être proposées à l'échelon de zones ou de pays.

\footnotetext{
* Intergovernmental Authority on Drought

** Southern African Development Community
} 


\section{DEFIN ITION DES GRANDES ZONES D'ECHANGE}

Les sept grandes zones d'échange retenues, représentées à la figure 1, sont :

- la zone O (Ouest). Elle est composée de la Mauritanie, du CapVert, du Sénégal, de la Gambie, de la Guinée-Bissau, de la Guinée, de la Sierra Leone et du Liberia. Cette zone se caractérise par peu d'échanges avec les autres zones (quelques courants entre le Mali et le Sénégal ou entre le Mali et la Guinée), par peu d'échanges entre les pays de la zone (hormis les ovins entre la Mauritanie et le Sénégal);

- la zone CO (Centre-Ouest). Elle comprend le Mali, le Burkina Faso, la Côte d'Ivoire, le Ghana et le Togo. Elle se caractérise par le pôle d'attraction pour les produits animaux constitué par la Côte d'Ivoire et, à un moindre degré, par le Ghana, le Togo servant de tampon avec la zone CN située à l'Est ;
- la zone CN (Centre-Nord). Elle inclut le Niger, le Tchad, la République centrafricaine, le Cameroun, le Bénin, et le Nigeria. Le Nigeria, et accessoirement le Cameroun, sont les pôles d'attraction des produits animaux. Le Bénin sert de tampon avec la zone CO. Quelques rares courants peuvent provenir de la zone $\mathrm{CO}$ à partir du Mali et du Burkina Faso. Quelques courants peuvent aussi exister avec les pays d'Afrique du Nord par des exportations du Niger et du Tchad. La République centrafricaine (Rca) et le Cameroun ont été rattachés à cette zone car la majorité des échanges est drainée par le Nigeria bien que l'on puisse noter des courants moins importants du Cameroun vers le Gabon et de la Rca vers le Congo ;

- la zone E (Est). Elle comprend le Soudan, l'Ethiopie, l'Erythrée, Djibouti, la Somalie, le Kenya et l'Ouganda. Cette zone correspond aux pays de l'Igad. Elle se caractérise par peu d'échanges avec les autres zones, une vocation à exporter vers la péninsule Arabique et marginalement vers l'Egypte pour la zone Nord du Soudan, ou vers l'Union européenne (Kenya) ;

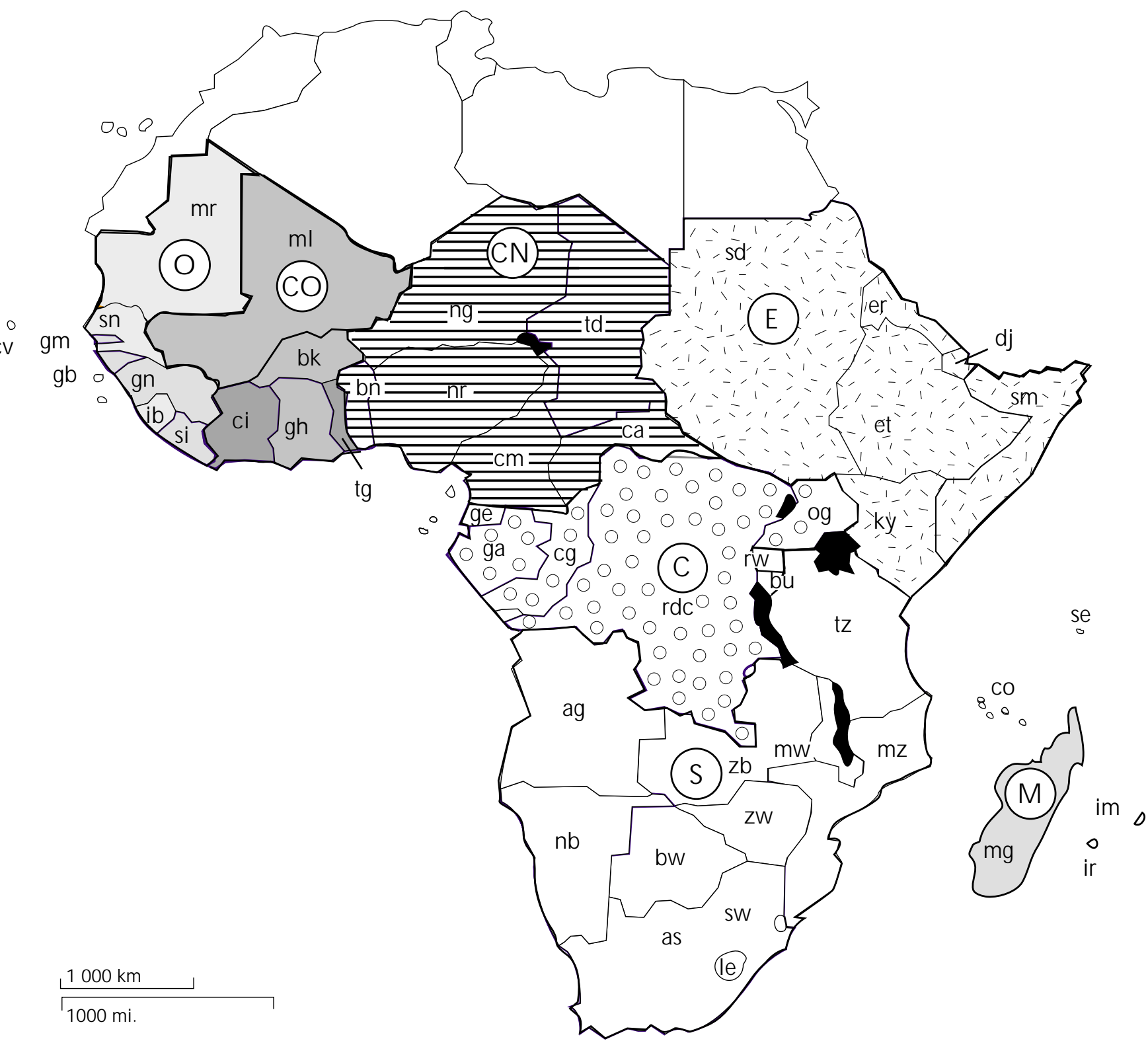

Figure 1 : zonage en sous-régions de l'Afrique subsaharienne en fonction des marchés, de l'homogénéité et de la complémentarité des échanges et en prenant en compte les unions politiques et économiques. $\mathrm{O}=$ zone $\mathrm{O}$ uest, $\mathrm{CO}=$ zone Centre- $\mathrm{O}$ uest, $\mathrm{CN}=\mathrm{zone}$ Centre-N ord, E = zone Est, C = zone Centre, S = zone Sud, M = zone Madagascar (Tacher G., Letenneur L., 1997, Cirad-emvt, M ontpellier, France, Bdpa-Scetagri, Paris, France). 
- la zone C (Centre). Elle est constituée du Gabon, du Congo, de la Guinée équatoriale, de Sao Tomé et Principe, de la République démocratique du Congo, du Rwanda et du Burundi. Sainte-Hélène a été ajoutée pour tout inclure. Cette zone se caractérise par peu d'échanges avec les autres zones. Elle est tournée vers l'extérieur du continent africain pour son approvisionnement en produits animaux ;

- la zone S (Sud). Elle correspond aux pays de la Sadc et comprend l'Angola, la Zambie, la Tanzanie, le Mozambique, le Zimbabwe, le Malawi, le Botswana, la Namibie, le Swaziland, le Lesotho, l'Afrique du Sud et l'Ile Maurice. Cette zone a beaucoup de courants intérieurs et peu d'échanges avec le reste du continent. Elle a des quotas d'exportation vers l'Union européenne. L'Ile Maurice a été rattachée à cette zone plutôt qu'à la zone $\mathrm{M}$ car elle appartient politiquement à la Sadc et elle a des échanges avec cette communauté ;

- la zone M (Madagascar). Zone de l'océan Indien, elle comprend Madagascar, La Réunion, les Comores et les Seychelles. Les échanges ont pour vocation de rester dans cette zone et, en fonction du disponible, de s'ouvrir en dehors de la zone. L'Ile Maurice est la liaison avec la zone $S$.

\section{ECHAN GES GLOBAUX DE L'ASS PAR ZONE}

Pour chaque zone, le calcul des soldes a été effectué à partir des importations et des exportations agrégées des pays qui les composent.

\section{Echanges en valeur de marchandises en Ass}

Le commerce des marchandises en Ass est relativement équilibré. Les importations s'élevaient en 1994 à 64,2 milliards de dollars américains courants et les exportations à 62,7 milliards et ont décuplé depuis 1964. Les zones $\mathrm{S}$ et $\mathrm{CN}$ réalisent respectivement 58 et 20 p. 100 des importations et 57 et 13 p. 100 des exportations. Les autres zones réalisent moins de 10 p. 100 du commerce subsaharien.

En Ass, le taux de couverture des importations par les exportations est de 103 p. 100 et varie dans le temps de 91 p. 100 en 1964 à 135 p. 100 en 1974. La couverture, de l'ordre de 100 p. 100 des importations, est le reflet de l'incapacité actuelle de ces nombreux pays à s'endetter plus et de l'obligation d'ajustement.

Les zones $\mathrm{CN}$ et $\mathrm{C}$ sont fortement exportatrices (taux de couverture de 160 p. 100), le solde des échanges des zones $\mathrm{CO}$ et $\mathrm{S}$ est équilibré et ceux des autres zones sont fortement déficitaires, en particulier la zone M qui importe cinq fois plus qu'elle n'exporte (figure 2).

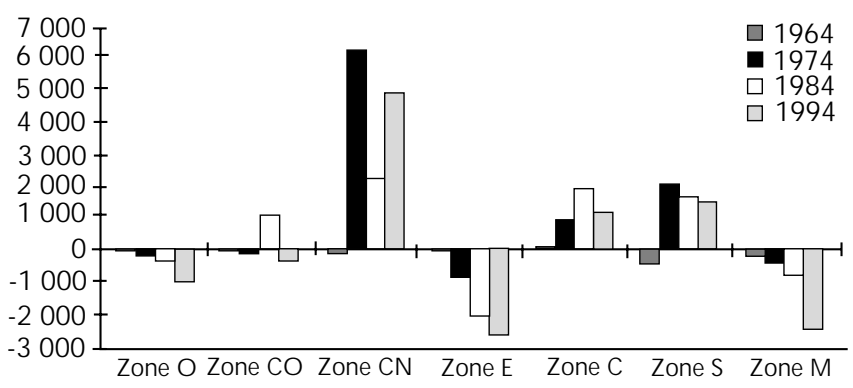

Figure 2 : soldes des échanges de marchandises en Ass (millions \$US). D'après la Fao 1995-1996.

\section{Commerce des produits agricoles par zone: la zone $S$ en réalise 40 p. 100}

L'Ass est exportatrice nette de produits agricoles, mais le solde excédentaire de ces échanges est en forte diminution. Les exportations représentent 129 p. 100 des importations, mais ce ratio est en diminution très forte sur le long terme, de 328 p. 100 en 1964 à 241 p. 100 en 1974 et à 141 p. 100 en 1984.

La zone $\mathrm{S}$ réalise 42 p. 100 des exportations de l'Ass et les zones $\mathrm{CO}$ et $\mathrm{E} 20$ p. 100. La zone $\mathrm{S}$ réalise 39 p. 100 des importations et les zones $\mathrm{O}, \mathrm{CN}$ et $\mathrm{E}$ environ 13 p. 100 chacune (figure 3). Le commerce des produits agricoles de la zone $\mathrm{CO}$ est très fortement excédentaire (taux de couverture de 300 p. 100), celui des zones E et $\mathrm{S}$ est aussi excédentaire (198 et 139 p. 100) mais en nette diminution depuis 30 ans. Toutes les autres zones étaient excédentaires en 1964 et sont fortement déficitaires aujourd'hui.

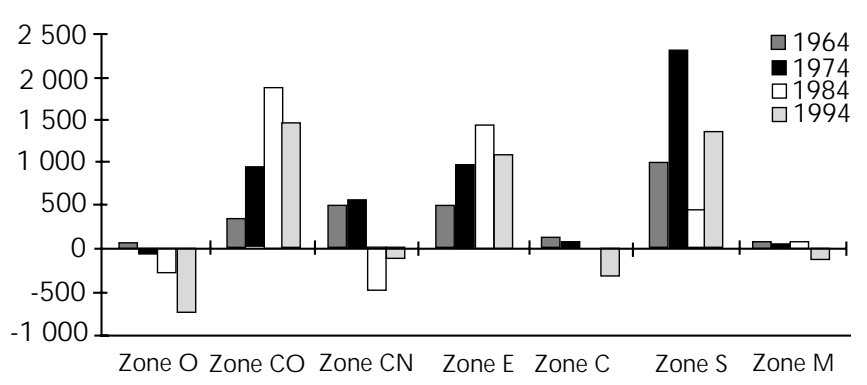

Figure 3 : soldes des échanges de produits agricoles en Ass (millions \$US). D'après la Fao 1995-1996.

\section{ECHANGES DE PRO DUITS ANIMAUX}

L'Ass est importatrice nette de produits animaux pour 590 millions de dollars, les importations représentent 1,6 fois les exportations. Les exportations de viandes représentent 71 p. 100 des échanges de produits animaux et celles des cuirs et des peaux 15 p. 100, les autres exportations sont négligeables sauf en zone $S$ (figure 4). Plus de 75 p. 100 des exportations de produits animaux des zones $\mathrm{O}, \mathrm{CO}, \mathrm{CN}, \mathrm{E}$ et $\mathrm{M}$ sont des viandes et le reste des cuirs et des peaux. Les produits laitiers, les œufs et les laines ne représentent rien.

La zone S exporte 47 p. 100 des viandes de l'Ass (soit 59 p. 100 de ses exportations de produits animaux), 87 p. 100 des produits laitiers et la totalité des œufs et des laines.

Les importations des produits animaux de l'Ass sont constituées pour 68 p. 100 par des viandes et 30 p. 100 par des produits laitiers.

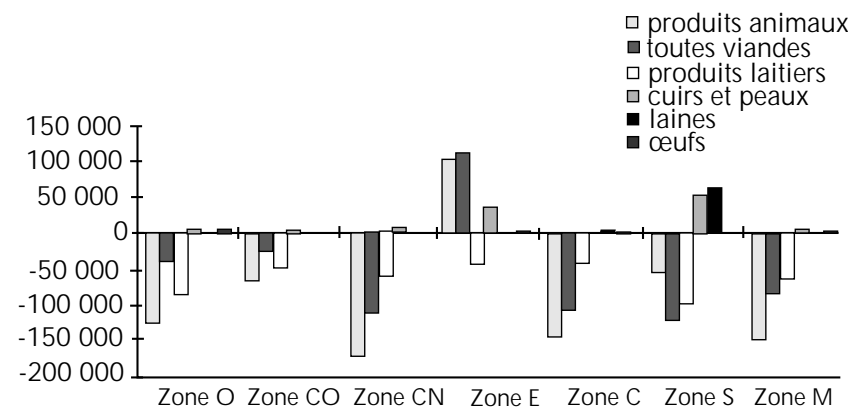

Figure 4 : soldes des échanges de produits animaux en Ass en 1994 (millions \$US). D’après la Fao 1995-1996. 
On peut distinguer trois types de zones dans la composition des importations : les zones $\mathrm{CO}, \mathrm{CN}, \mathrm{C}$ et $\mathrm{S}$ qui importent en gros trois quarts de viande et un quart de lait de l'Afrique subsaharienne, les zones $\mathrm{O}$ et $\mathrm{M}$ qui importent autant de lait que de viande et la zone E qui est presque exclusivement importatrice de lait (figure 5).

En Ass, le solde commercial des laines et des cuirs est excédentaire de 207 millions de dollars et celui des viandes et des produits laitiers est déficitaire respectivement de 366 et 428 millions de dollars. Pour ce qui concerne les échanges de viandes, seule la zone E (Igad) connaît un excédent.

Les pourcentages des sept sous-régions pour les importations et exportations de l'Ass figurent dans le tableau I.

Le commerce des viandes est ici pris au sens le plus large. Il comprend le commerce des viandes, des préparations diverses et des animaux vivants qui sont soit abattus directement soit engraissés en feed lot avant abattage. Le solde des échanges de viandes de l'Ass se dégrade. Il est déficitaire de 366 millions de dollars après avoir été excédentaire pendant les années 1960-70. En 30 ans, les importations ont été multipliées par 6,5 et atteignent 1064 millions de dollars en 1994, alors que les exportations n'ont été multipliées que par 2,5 pour atteindre 698 millions de dollars. Les exportations de bovins représentent 67 p. 100 et celles de petits ruminants 31 p. 100 des viandes. Les exportations de porcs, de volailles et d'autres espèces sont négligeables.

Les exportations des zones $\mathrm{C}$ et $\mathrm{M}$ sont quasi nulles et celles de la zone $\mathrm{O}$ négligeables. Les exportations de la zone $\mathrm{S}$ sont constituées à 85 p. 100 de bovins et à 12 p. 100 de petits ruminants. La zone $\mathrm{S}$ est aussi le principal exportateur de viande de porc (69 p. 100 de l'Ass), de viande de volaille et d'autres espèces (environ 90 p. 100). Les zones $\mathrm{CN}$ et $\mathrm{CO}$ (respectivement 16 et 14 p. 100 de l'Ass) sont surtout exportatrices de bovins (77 et

\section{Tableau I}

Importations et exportations toutes viandes par zones de l'Ass (\% des valeurs totales de l'Ass)

\begin{tabular}{llllrrrr} 
& O & CO & CN & E & C & S & M \\
\hline Importations & 7 & 11 & 21 & 0 & 10 & 43 & 8 \\
Exportations & 6 & 14 & 16 & 16 & 0 & 47 & 1
\end{tabular}

62 p. 100 de leurs exportations de viande) et de petits ruminants pour le solde. La zone E (16 p. 100 de l'Ass) est exportatrice de petits ruminants $(88$ p. 100).

La zone Igad n'importe pas de viande. Les importations de viandes bovines devancent celles des petits ruminants (sauf en zone $\mathrm{O}$ ), des volailles et des porcs. En deuxième position, les zones $\mathrm{CO}, \mathrm{CN}$ et $\mathrm{S}$ importent des petits ruminants, les zones $\mathrm{M}$ et $\mathrm{E}$ du porc et la zone $\mathrm{C}$ de la volaille. Les plus gros importateurs en valeur de viande de bovin sont les zones $\mathrm{S}$ avec 202 millions de dollars (202 M\$) et CN avec $172 \mathrm{M} \$$. Les plus gros importateurs de petits ruminants sont les zones $\mathrm{S}(115 \mathrm{M} \$)$, $\mathrm{CN}(44 \mathrm{M} \$)$ et $\mathrm{O}(39 \mathrm{M} \$)$. Les plus gros importateurs de viande de volaille sont les zones $\mathrm{S}$ (88 M\$) et C (40 M\$). Les plus gros importateurs de viande de porc sont les zones $\mathrm{S}(43 \mathrm{M} \$)$ et $\mathrm{M}(17 \mathrm{M} \$)$.

La part de ces différentes viandes dans les importations a fortement évolué au cours des 30 dernières années (figure 6). Les importations de viande de bœuf diminuent assez fortement dans l'ensemble des zones au profit de la volaille, des petits ruminants et un peu du porc. Les autres espèces restent marginales. La part des importations de viande de petit ruminant a augmenté fortement en zones $\mathrm{O}, \mathrm{CN}$ et $\mathrm{S}$ et a stagné ou régressé dans les autres zones. Les importations relatives de viande de volaille explosent dans les zones CO, C, S et M. La situation du porc est plus contrastée, son évolution lente est surtout le fait des zones M, dont les importations relatives diminuent, et $\mathrm{S}$ dont les importations ont tendance à augmenter.

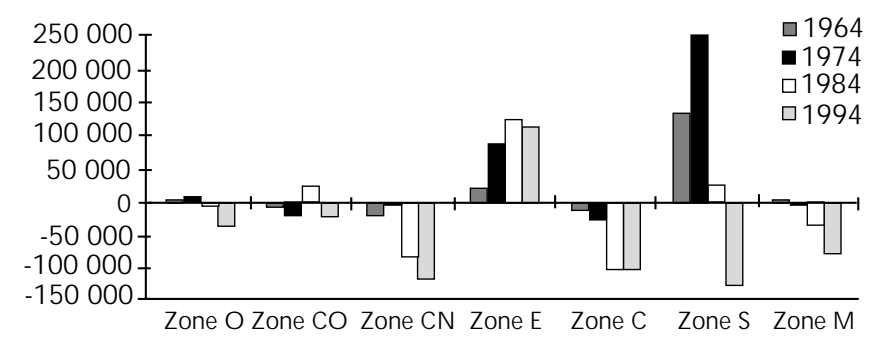

Figure 6 : évolution des soldes des échanges toutes viandes en Ass (1 000 \$U S). D'après la Fao 1995-1996.

\section{ECHAN GES PAR PRODU CTION DES ZONES DE L'ASS}

\section{Viande bovine : échanges excédentaires pour l'Igad et l'Afrique australe}

Le commerce de la viande bovine comprend les échanges d'animaux vivants, de viandes réfrigérées ou congelées, d'abats et de préparations (extraits, viandes en boîte). Les pourcentages des sept sous-régions pour les importations et exportations de viande bovine de l'Ass figurent dans le tableau II.

\section{Tableau II}

Importations et exportations de viande bovine par zones de l'Ass (\% des valeurs totales de l'Ass)

$\begin{array}{llllllll} & \text { O } & \text { CO } & \text { CN } & \text { E } & \text { C } & \text { S } & \text { M } \\ \text { Importations } & 6 & 14 & 29 & 0 & 9 & 35 & 7 \\ \text { Exportations } & 5 & 13 & 19 & 3 & 0 & 59 & 1\end{array}$


Globalement, l'Ass est importatrice nette de 120 millions de dollars de viande bovine en 1994 après avoir été exportatrice jusque dans les années 80 . Les zones $\mathrm{CO}, \mathrm{CN}$ et $\mathrm{S}$ sont à la fois les grandes zones exportatrices et importatrices. Les zones $\mathrm{CO}, \mathrm{CN}$ et $\mathrm{C}$ sont historiquement déficitaires en viande bovine et leurs déficits, proportionnellement à l'ensemble de l'Ass, ont été multipliés par 3 ou 4 entre 1964 et 1994. Les zones O et M sont devenues déficitaires. L'Igad, malgré un troupeau important, est faiblement excédentaire et les exportations de la zone $\mathrm{S}$ ont diminué de 40 p. 100 en dollars courants en 30 ans (figure 7).

Les exportations des zones $\mathrm{O}, \mathrm{CO}$ et $\mathrm{CN}$ sont constituées exclusivement de bovins vivants importés par les pays consommateurs de la côte à partir des pays éleveurs sahéliens. Les exportations de la zone $\mathrm{S}$ sont très différentes, constituées à $70 \mathrm{p}$. 100 de viandes et de préparations et à 29 p. 100 d'animaux vivants. L'appareil industriel mis aux normes internationales, bien dimensionné, permet une bonne valorisation de l'élevage des ranchs de la zone australe. Les exportations intra-zones de Namibie, du Zimbabwe et du Botswana en direction de l'Afrique du Sud sont réalisées aussi bien sous forme d'animaux maigres à engraisser que de viandes congelées. Les exportations hors Ass à partir des pays cités précédemment et de l'Afrique du Sud sont réalisées sous forme de viandes souvent découpées et de préparations.

Les importations des zones $\mathrm{CO}$ et $\mathrm{CN}$ sont réalisées en vif (respectivement 75 et 97 p. 100 du bœuf importé) à partir des pays sahéliens. La zone $\mathrm{O}$ importe aussi 61 p. 100 de bovins vivants mais elle importe le solde en viandes et en préparations. A l'opposé, 99 p. 100 des importations des zones $\mathrm{C}$ et $\mathrm{M}$ sont des viandes, des abats et des préparations. Le commerce des bovins en zone $\mathrm{S}$ est équilibré comme nous l'avons vu précédemment.

Le solde de ces échanges permet de déduire de l'agrégation des importations et des exportations les échanges de chaque zone et de l'Ass avec l'extérieur. Les zones $\mathrm{CO}, \mathrm{C}$ et $\mathrm{M}$ sont importatrices nettes de viandes et d'abats à partir de l'extérieur (Europe, Usa...) et d'Afrique australe (pour la zone M). La zone $\mathrm{O}$ est de plus en plus importatrice de viande et d'abats de bœuf et elle est traditionnellement exportatrice de bovins vivants, en particulier en direction de la zone $\mathrm{CN}$. La zone E échange peu, après avoir été historiquement exportatrice de viande et d'animaux vivants. La zone $\mathrm{S}$ reste exportatrice nette pour environ 90 millions de dollars de produits à haute valeur ajoutée et importatrice pour 15 millions de dollars d'abats et de bovins vivants après avoir été historiquement exportatrice de tous les produits bovins jusque dans les années 80 .

Aujourd'hui, l'Ass est donc importatrice nette de 73 millions de dollars d'animaux vivants et de 47 millions de dollars de viandes (répartis de manière à peu près égale : 15 millions de viandes, 19 millions d'abats et 14 millions de préparations de viandes). Bien qu'exportatrice nette de 55000 bovins vivants, l'Ass est défi-

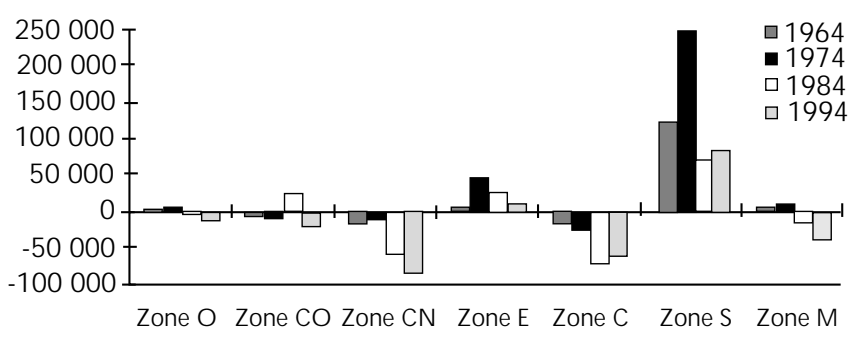

Figure 7 : évolution des soldes des échanges toute viande bovine en Ass (1 000 \$US). D'après la Fao 1995-1996. citaire en valeur par suite d'une comptabilisation en Fob (free on board) pour les exportations à 432 dollars par bovin et en Caf (coût assurance fret) pour les importations à 315 dollars par bovin, soit une différence de 27 p. 100.

\section{Petits ruminants : Igad, premier exportateur de l'Ass}

En Ass, le commerce des petits ruminants représentait, en 1994, environ 220 millions de dollars et le solde était faiblement déficitaire (6 millions). Historiquement, l'Ass a été largement excédentaire (95 millions de dollars en 1984).

Les grandes zones d'exportation sont l'Igad (46 p. 100 en valeur) et les zones CO (17 p. 100) et S (17 p. 100). Les grandes zones importatrices sont les zones $\mathrm{O}$ (17 p. 100 en valeur), $\mathrm{CN}$ (20 p. 100) et $S$ (51 p. 100). Au total, l'Igad est très excédentaire (100 millions), la zone $S$ très déficitaire ( 77 millions), la zone $\mathrm{CO}$ excédentaire (15 millions), ainsi que les zones $\mathrm{O}$ et $\mathrm{CN}$ (respectivement 20 et 18 millions de dollars).

Les exportations sont généralement réalisées en vif (97 p. 100) sauf dans la zone $\mathrm{M}$ qui reste très marginale. Par contre, 63 p. 100 des petits ruminants sont importés vivants et 37 p. 100 sous forme de viande à partir de pays à l'extérieur de l'Afrique (NouvelleZélande, etc.).

Les zones importatrices d'animaux vifs sont les zones $\mathrm{O}, \mathrm{CO}, \mathrm{CN}$ et $\mathrm{S}$. Cette dernière réalise la quasi-totalité des importations de viande d'ovins et de caprins (figure 8). Depuis 1964, l'Ass est globalement exportatrice vers l'Afrique du Nord et la péninsule Arabique de petits ruminants vivants (70 M\$ en 1994) et de plus en plus importatrice de viande (76 M\$ en 1994). L'Igad est exportateur net de petits ruminants vivants et la zone $S$ importatrice nette de viande. La zone $\mathrm{CO}$ exporte vers les autres zones (surtout $\mathrm{O}$ et $\mathrm{CN})$ des animaux vivants $(17 \mathrm{M} \$)$ et les zones $\mathrm{O}$ et $\mathrm{CN}$ sont importatrices d'animaux vivants (respectivement 20 et $18 \mathrm{M} \$$ ).

\section{Echanges de viande de porc : marché d'importation à 95 p. 100}

Le solde de l'Ass était déficitaire en viande de porc de 77 millions de dollars en 1994 et ce déficit s'est amplifié depuis 30 ans. Les plus gros importateurs sont les zones $\mathrm{S}$ (52 p. 100 en valeur) et $\mathrm{CN}$ (28 p. 100). Depuis 1964, toutes les zones de l'Ass sont déficitaires ou très faiblement excédentaires et s'approvisionnent sur les marchés en dehors de l'Afrique. Les zones $\mathrm{O}, \mathrm{CO}$ et $\mathrm{CN}$ importent cependant de moins en moins de porc. Leur participation au déficit de l'Ass s'est ainsi divisée respectivement par 6, 7 et 14 en 30 ans. Les exportations de l'Ass sont négligeables (4 M\$) et proviennent des zones S (70 p. 100 en valeur) et E (29 p. 100) autant sous forme de viande que de préparations (bacon, jambon, viande séchée et saucisses).

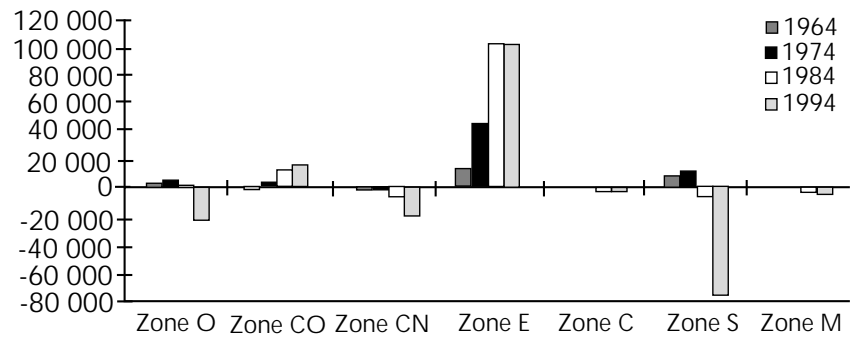

Figure 8 : évolution des soldes des échanges toute viande de petit ruminant en Ass (1 000 \$U S). D'après la Fao 1995-1996. 
Les importations de 81 millions de dollars sont réalisées principalement par les zones $\mathrm{C}, \mathrm{S}$ et $\mathrm{M}$. Les importations des zones S et M sont en forte augmentation (figure 9). La composition des importations est variable. Les zones faiblement importatrices, $\mathrm{O}, \mathrm{CO}, \mathrm{CN}$ et $\mathrm{E}$, achètent surtout des préparations à base de porc, les zones $\mathrm{C}$ et $\mathrm{M}$ importent plutôt de la viande et la zone $\mathrm{S}$ importe 39 p. 100 de viandes, 55 p. 100 de préparations et 6 p. 100 de porcs vivants. Depuis 1964, la part relative de la viande dans les importations de porc a triplé au détriment du commerce en vif et des préparations.

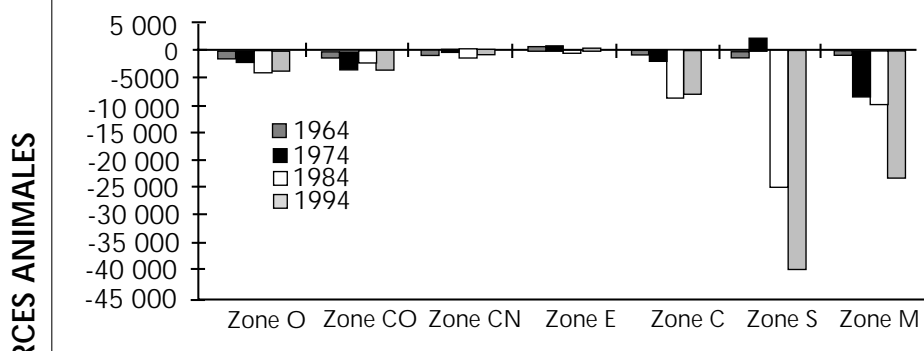

Figure 9 : évolution des soldes des échanges toute viande de porc en Ass (1 000 \$US). D'après la Fao 1995-1996.

\section{Viande de volaille : marché d'importation}

Ce commerce est difficile à estimer sauf, peut-être, en ce qui concerne les importations d'origine européenne ou américaine. Les volailles produites en Afrique sont très peu exportées. La zone $\mathrm{S}$ est seule exportatrice de quantités infimes (4 M\$). Toutes les zones achètent de la volaille hors Ass pour un montant global de 164 millions de dollars. Les zones C ( 23 p. 100 en valeur) et $S$ (53 p. 100) sont les principales importatrices (figure 10). Ce marché s'est multiplié par 110 en 30 ans, en dollars courants, et consiste principalement à acheter les résidus de découpe des industries occidentales (ailerons, croupions, poules de réforme...).

\section{Lait et produits laitiers}

Toutes les zones de l'Ass sont structurellement déficitaires en produits laitiers et le déficit global s'élève à 428 millions de dollars. Les exportations (46 M\$) ne représentent pas 10 p. 100 des importations. Aujourd'hui, la zone S réalise 87 p. 100 de ces ventes et l'Igad seulement 11 p. 100, alors que cette dernière était la principale zone de vente de produits laitiers de l'Ass jusqu'à la fin des années 70 (figure 11). Les exportations de lait (frais, en poudre, concentré ou petit-lait concentré) équivalent à 88 p. 100 du total, celles de beurre et de fromage respectivement à 8 et 4 p. 100. En 1964, les exportations de beurre et d'huile de beurre (ghee) constituaient l'essentiel (60 p. 100) des ventes de l'Ass, mais la situation s'est rapidement inversée.

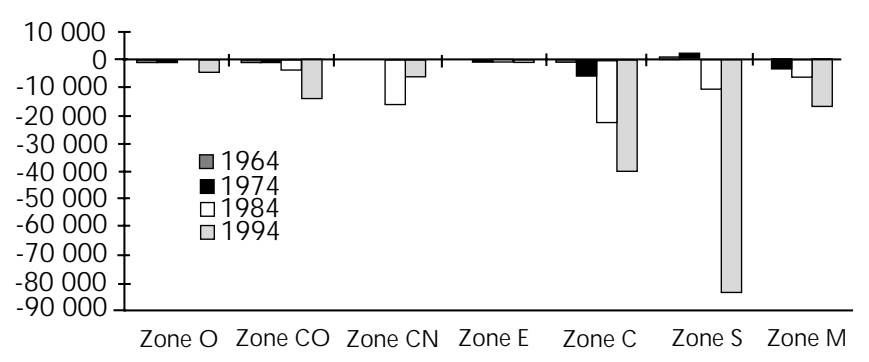

Figure 10 : évolution des soldes des échanges toute viande de volaille en Ass (1 000 \$US). D'après la Fao 1995-1996.
Les importations de l'Ass s'élèvent à 474 millions de dollars et sont à 90 p. 100 d'origine extérieure à l'Afrique. Toutes les zones importent de fortes quantités de lait. En valeurs, les proportions des importations sont de 29 p. 100 pour la zone S, 17 p. 100 pour la zone $\mathrm{O}, 14$ p. 100 pour la zone $\mathrm{CN}, 13$ p. 100 pour la zone $\mathrm{M}$, 10 p. 100 pour les zones $\mathrm{CO}$ et $\mathrm{E}$ et 7 p. 100 pour la zone C. Les laits représentent 80 p. 100 de la valeur des importations en produits laitiers soit 377 millions de dollars pour l'année 1994, et les beurres et les fromages représentent respectivement 9 et 11 p. 100. Depuis 1964, la part relative du lait dans les importations a augmenté de 14 p. 100 au détriment de celle du beurre, alors que celle des fromages est restée stable. La zone M fait exception car ses importations de beurre et de fromage représentent la majeure partie (respectivement 38 et 16 p. 100) des produits laitiers achetés.

\section{Les æufs}

La seule région exportatrice de l'Ass est la zone $\mathrm{S}$ dont les ventes agrégées s'élèvent à 9,5 millions de dollars. Les importations globales se montent à 12,3 millions de dollars dont 40 p. 100 pour la zone $\mathrm{S}$. La zone $\mathrm{S}$ a un solde excédentaire de 4,6 millions de dollars et toutes les autres zones sont déficitaires, en particulier la zone $\mathrm{O}$ avec un déficit de 3,4 millions de dollars et les zones $\mathrm{C}$, $\mathrm{E}$ et $\mathrm{M}$ avec environ 1 million de dollars. En Ass, le commerce des œufs est généralement réalisé en coquille, seule la zone $\mathrm{S}$ exporte 14 p. 100 de ses œufs (1,35 M\$) sous forme liquide ou en poudre.

\section{Echanges de laine : marché d'exportation de l'Afrique australe}

Les exportations de laine représentent 8 p. 100 des exportations de l'Ass dont une grande partie est sous forme de devises. Les secteurs de la laine, des cuirs et des peaux sont les seuls secteurs excédentaires des produits animaux en Ass. L'exportation nette de laine a rapporté 62 millions de dollars en 1994. L'Afrique australe réalise la totalité du commerce de l'Ass avec 80,1 millions de dollars d'exportations et 18 millions de dollars d'importations. Cet excédent commercial est réalisé à l'extérieur du continent. La chute des prix internationaux de la laine a pratiquement induit la faillite de l'économie lainière et la disparition de l'élevage du mouton Karakul.

\section{Echanges des cuirs et des peaux : marché d'exportation de toutes les zones de l'Ass}

Bien que souvent exportés sous forme de produits bruts ou semifinis, les cuirs et les peaux représentent 147,5 millions de dollars, soit 15 p. 100 des exportations de l'Ass. La zone S exporte pour 99 millions de dollars de cuirs et de peaux (67 p. 100 des ventes) et l'Igad 33 millions (23 p. 100). Les ventes des autres zones sont marginales. Quelques importations (2,5 M\$) sont réalisées principalement dans la zone $\mathrm{S}$. Les cuirs et les peaux vendus en grande

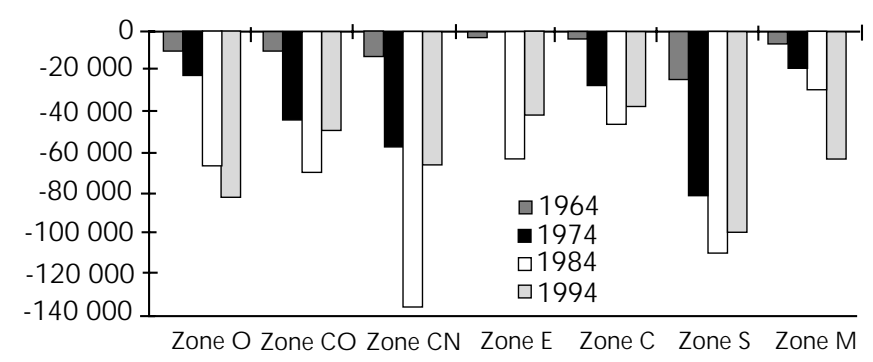

Figure 11 : évolution des soldes des échanges de produits laitiers en Ass (1 000 \$US). D'après la Fao 1995-1996. 
partie à l'extérieur de l'Ass représentent ainsi un revenu de 145 millions de dollars. Ce solde positif compense presque les importations nettes de volailles (164 M\$) ou le double des importations de porc (77 M\$) ou encore les importations nettes de bœuf.

\section{CON SOMMATION SELON LES ZONES}

L'analyse par zone des consommations de viande seules permet de distinguer trois modèles de consommation (figure 12) :

- un type de consommation basée sur la viande de bœuf ; les populations des zones E, $\mathrm{S}$ et $\mathrm{M}$ consomment 43 p. 100 de viande bovine pour la première et 47 p. 100 pour les deux autres zones. De façon plus précise, la consommation est essentiellement centrée sur le bœuf et les petits ruminants en zone E, et sur le bœuf et les volailles en zones $\mathrm{S}$ et $\mathrm{M}$;

- un type de consommation équilibré observé dans les zones $\mathrm{C}, \mathrm{CO}$ et $\mathrm{CN}$ avec une alimentation en viande variée. La consommation de viande de porc reste cependant faible en zone $\mathrm{O}$ et $\mathrm{CO}$ (inférieure à 10 p. 100) ;

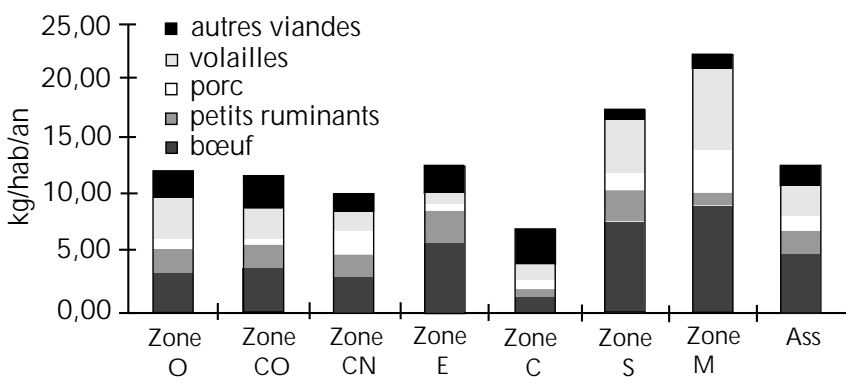

Figure 12 : quantité de viandes consommées par zone (kg/hab/an).
- un type gibier en zone $\mathrm{C}$ où il constitue la principale source de viande dans cette région de savanes et de forêt.

Si l'ensemble des produits d'origine animale est considéré, il se dégage quatre modèles de consommation :

- un type équilibré dans les zones $\mathrm{CN}, \mathrm{S}$ et $\mathrm{M}$, en particulier dans la zone $\mathrm{CN}$. Dans les zones $\mathrm{S}$ et $\mathrm{M}$, les divers produits sont en premier les viandes rouges, puis le lait et les volailles, enfin le poisson ;

- un type «poisson-viande » dans les zones côtières $\mathrm{O}$ et $\mathrm{CO}$, dans lequel le poisson contribue pour 36 p. 100 aux apports protéiques, soit plus que toute espèce animale élevée ;

- un type « poisson-gibier » en zone $\mathrm{C}$ où la viande provient principalement de prélèvements dans le milieu naturel (1) ;

- un type lait en zone $\mathrm{E}$ où le lait est la première source protéique.

Au-delà de ces types, les chiffres montrent également dans le tableau III des niveaux de consommation très variables selon les zones. Il met en évidence une hétérogénéité régionale considérable dans la consommation journalière des protéines, allant de 4,2 à 13,8 g par habitant, autour d'un modèle agrégé représentant l'Afrique subsaharienne, avec une moyenne de 9,2 g. Si l'on désagrège davantage, les disparités dans la consommation deviennent encore plus grandes, d'abord entre pays, puis entre zones rurales et urbaines et en fonction des revenus.

Les disparités sont également grandes entre les différents modèles de consommation des protéines animales comme l'ont montré les types ci-dessus. Dans la zone des pays de l'Igad (zone E), la consommation de produits laitiers est dominante et atteint $7,2 \mathrm{~g} / \mathrm{hab} / \mathrm{j}$. Elle est négligeable dans la zone $\mathrm{C}$ avec $0,4 \mathrm{~g} / \mathrm{hab} / \mathrm{j}$. La proportion de protéines de viande bovine ne varie que du simple au double selon les zones. Il en est de même pour la viande de petits ruminants (à l'exception de Madagascar). Enfin dans la zone C, dominée par la République démocratique du Congo, le gibier représente 37 p. 100 des protéines animales consommées (1) alors que dans la zone M, dominée par Madagascar, la consommation n'est que de 2 p. 100 des protéines animales de la ration.

\section{Tableau III}

Consommation en protéines animales pour chaque zone dans les sept sous-régions : selon leur nature en pourcentages de la consommation totale, selon la consommation totale en tonnes par jour et selon la consommation journalière en grammes par habitant

$\begin{array}{lrrrrrrrrr}\text { Produits } & \text { E } & \text { M } & \text { S } & \text { O } & \text { CO } & \text { CN } & \text { C } & \text { Ass } & \text { Variabilité * } \\ \text { Bovins } & 22 & 31 & 33 & 16 & 24 & 23 & 19 & 21 & 2: \text { très faible } \\ \text { Petits ruminants } & 10 & 2 & 9 & 11 & 13 & 14 & 5 & 10 & 2,8: \text { faible (hors M) } \\ \text { Porcs } & 1 & 14 & 6 & 5 & 6 & 15 & 13 & 6 & 15: \text { très forte } \\ \text { Volailles } & 5 & 22 & 18 & 17 & 15 & 13 & 17 & 13 & 4,4: \text { forte } \\ \text { Gibiers } & 7 & 2 & 3 & 8 & 18 & 9 & 37 & 8 & \text { peu significative } \\ \text { Abats/conserves } & 0 & 2 & 1 & 1 & 1 & 0 & 0 & 5 & \text { non significative } \\ \text { Laits } & 52 & 24 & 24 & 34 & 19 & 12 & 9 & 32 & 5,8: \text { forte } \\ \text { Fufs } & 2 & 3 & 6 & 6 & 4 & 13 & 1 & 5 & 13: \text { très forte } \\ \text { Protéines consommées } & 1952 & 203 & 1470 & 227 & 380 & 850 & 254 & 5336 & \\ \text { (tonnes/jour) } & & & & & & & & \end{array}$

* Rapport de la zone de consommation maximale à la zone de consommation minimale 
L'analyse des évolutions des consommations par habitant (kg/an) de1964 à 1994 est rapportée dans le tableau IV.

Ces chiffres montrent une baisse importante et relativement homogène des consommations de viandes rouges, à l'exception des zones $\mathrm{CO}$ et $\mathrm{CN}$ où la baisse de consommation est moindre. Il en est de même pour le lait avec une plus grande disparité selon les zones. Seules les zones E, forte consommatrice de lait, et $\mathrm{C}$, à l'opposé très peu consommatrice, ont peu modifié les quantités consommées. En revanche, une forte augmentation des viandes blanches est observée dans toutes les sous-régions, avec des quantités consommées qui ont doublé et même triplé dans les zones $\mathrm{O}$ et $\mathrm{CN}$ et qui ont atteint des quantités voisines de celles des viandes rouges en zones $\mathrm{O}$ et $\mathrm{M}$. On assiste donc à une modification importante des modes de consommation dans la plupart des zones. La consommation d'œufs a été multipliée par 1,5-2,4 entre 1964 et 1994 dans quatre zones avec des consommations comprises entre 1,2 et $3,2 \mathrm{~kg}$, à l'exception des zones $\mathrm{E}$ (- 14 p. 100), C (très faible consommation : $0,3 \mathrm{~kg}$ ) et $\mathrm{M}$ (pas de variations).

\section{L'OFFRE}

L'offre est également très variable selon les zones. Les effectifs des différentes espèces animales sont fort différents d'une zone à l'autre comme le montrent les données du tableau V. Les densités humaines sont dans une fourchette limitée (variation de 1 à 2 entre les extrêmes), mais les unités de bétail tropical (Ubt) et les volailles rapportées par habitant sont beaucoup plus variables. Ces données reflètent les tendances rapportées dans les consommations.

En ce qui concerne la production de viande bovine, malgré des effectifs importants, la faiblesse de la productivité des troupeaux est une caractéristique majeure de ce secteur et se situe autour de $15,75 \mathrm{~kg} /$ animal/an pour toutes les zones. Elle est cependant réalisée avec très peu d'intrants ce qui assure une certaine compétitivité dans les prix. Les productions des troupeaux de petits ruminants sont également faibles avec une productivité pondérale de $3,66 \mathrm{~kg} / \mathrm{animal} / \mathrm{an}$. Cette production augmente de 1,9 p. 100 par an avec de très fortes variations entre les zones : taux de croissance

\section{Tableau IV}

Evolution des consommations des principaux groupes de produits d'origine animale dans les sept régions de l'Ass (kg/hab/an)

\begin{tabular}{|c|c|c|c|c|c|c|c|c|c|}
\hline \multirow[t]{2}{*}{ Zone } & \multicolumn{3}{|c|}{ Viandes rouges } & \multicolumn{3}{|c|}{ Viandes blanches } & \multicolumn{3}{|c|}{ Lait } \\
\hline & 1964 & 1994 & $\%$ & 1964 & 1994 & $\%$ & 1964 & 1994 & $\%$ \\
\hline 0 & 7,32 & 5,4 & $-26,2$ & 1,6 & 4,78 & +199 & 33,95 & 29,33 & $-13,6$ \\
\hline $\mathrm{CO}$ & 6,48 & 5,84 & $-10,2$ & 1,82 & 3,2 & +75 & 20,01 & 13,36 & $-33,2$ \\
\hline $\mathrm{CN}$ & 5,12 & 5,09 & $-0,6$ & 1,33 & 3,81 & +186 & 11,62 & 9,52 & $-18,1$ \\
\hline$E$ & 13,66 & 8,81 & $-35,5$ & 1,4 & 1,73 & +23 & 74,07 & 71,53 & $-3,4$ \\
\hline C & 2,36 & 1,83 & $-22,5$ & 1,3 & 2,33 & +79 & 3,78 & 3,76 & $-0,5$ \\
\hline$S$ & 14,78 & 10,51 & $-28,9$ & 3,04 & 6,14 & +102 & 64,29 & 35,59 & $-44,6$ \\
\hline M & 16,25 & 10,21 & $-37,2$ & 7,73 & 10,86 & +40 & 54,08 & 37,23 & $-31,2$ \\
\hline
\end{tabular}

Tableau V

Effectifs des cheptels par zone, population humaine et quelques indicateurs par rapport au disponible par habitant

\begin{tabular}{|c|c|c|c|c|c|c|c|c|}
\hline Cheptel (x 1 000) & 0 & $\mathrm{CO}$ & CN & $\mathbf{E}$ & C & $\mathbf{S}$ & M & Total \\
\hline Bovins & 6969 & 12962 & 31733 & 75330 & 2466 & 45226 & 10366 & 185076 \\
\hline O vins & 11247 & 16596 & 24714 & 66840 & 1999 & 38371 & 757 & 160532 \\
\hline Caprins & 8326 & 20837 & 39534 & 57757 & 6485 & 26810 & 1465 & 161061 \\
\hline Porcs & 1361 & 2462 & 9461 & 1041 & 1591 & 3735 & 1658 & 21279 \\
\hline Volailles & 69000 & 87000 & 189000 & 144000 & 45000 & 159000 & 32000 & 716000 \\
\hline Population humaine (x 1000 ) & 26674 & 55243 & 144849 & 141841 & 60832 & 135518 & 15648 & 580605 \\
\hline Superficie (x 1000 km²) & 1760 & 2133 & 4683 & 5207 & 3038 & 6891 & 592 & \\
\hline Densité & 15,2 & 25,9 & 30,9 & 27,2 & 20 & 19,7 & 26,4 & \\
\hline Ubt/hab & 0,32 & 0,44 & 0,24 & 0,56 & 0,05 & 0,34 & 0,55 & \\
\hline Volaille/hab & 2,59 & 1,57 & 1,30 & 1,02 & 0,74 & 1,17 & 2,05 & \\
\hline
\end{tabular}


allant de 1,1 p. 100 en zones E et S, à 4,9 p. 100 en zone CN. Les effectifs de porcs ont connu une croissance de 4,2 p. 100 sur l'ensemble des zones. La production de volailles est le secteur qui a enregistré la plus forte croissance annuelle depuis 30 ans, avec 5,2 p. 100 par an, allant de 3 p. 100 en zone E à 7,4 p. 100 en zone S. La production de lait a été limitée à 2 p. 100 par an avec de faibles variations selon les zones. La faiblesse de la productivité du cheptel est encore plus nette que pour la viande.

A côté de ces productions doivent s'ajouter les importations. Pour les bovins sur pied, seule la zone $\mathrm{CN}$ est importatrice nette s'approvisionnant dans la zone $\mathrm{CO}$, seule zone réellement exportatrice. Toutefois, ces échanges restent limités. Il en est de même pour les petits ruminants pour lesquels la zone $\mathrm{CN}$ a un solde très déficitaire. Toutes les sous-régions sont importatrices en viandes, à l'exception de la zone E. Les zones C et S importent le plus en 1994 avec respectivement 86000 et 239000 tonnes, soit 80 p. 100 des importations de l'Ass en viandes. Ce sont principalement les viandes de bovin et de volaille qui sont importées, avec un véritable bond pour ces dernières depuis 15 ans qui atteignent 150000 tonnes équivalent carcasse (tec) en 1994, en majorité dans les zones $\mathrm{E}$ et $\mathrm{S}$. Toutes les zones importent massivement tous les produits laitiers, à l'exception là encore de la zone E. Dans les zones $\mathrm{C}$ et $\mathrm{S}$, les importations représentent près de 50 p. 100 de la production, dans les zones $\mathrm{CO}$ et $\mathrm{M}$ environ 20 p. 100 et dans la zone $\mathrm{CN} 13$ p. 100. La poudre de lait représente 69 p. 100 des importations de produits laitiers de l'Ass avec 855000 tonnes équivalent lait. Ces importations ne compensent pas les quantités nécessaires pour assurer un apport constant en protéines animales aux populations des différentes sous-régions identifiées dont la consommation diminue d'année en année.

\section{CONCLUSION}

Ce zonage de l'Ass en sous-régions donne des résultats cohérents avec des entités géographiques homogènes de productions et d'échanges de produits animaux à l'intérieur de chaque zone. Ce bilan des échanges montre également une grande diversité des sept zones définies dans les productions, les parts des marchés et les quantités de protéines animales consommées. Ces disparités sont également importantes dans les types de consommation.

Les marchés sont dominés par les échanges en viande bovine et en lait. Toutefois, les viandes blanches, en particulier les viandes de volaille, prennent une part de plus en plus importante dans les productions animales et les importations. On observe, par ailleurs, la part prépondérante de l'Afrique australe (zone S) dans les échanges.

Au-delà des productions traditionnelles obtenues en conduite extensive des troupeaux, des productions selon des modes de conduite intensive se développent en particulier pour les monogastriques et, dans une moindre mesure à ce jour, pour le lait. Cette évolution tient en grande partie à l'urbanisation qui engendre une forte pression sur l'offre.

S'il y a quelques traits communs pour le développement des productions animales en Ass, cette grande diversité amène à proposer des voies différentes selon les sous-régions. Ce sera l'objet de la troisième partie de cette étude qui contribuera à identifier les voies les mieux adaptées pour satisfaire les demandes dans les sept sousrégions de l'Ass.

\section{BIBLIO GRAPHIE}

1. CHARDO N NET PH. Ed., 1995. Faune sauvage africaine : la ressource oubliée, tomes I et II. Luxembourg, Bruxelles, Belgique, Ceca/CE/Ceea, 416 p., 288 p.

2. CFCE, 1991. Le marché des produits laitiers de base dans 58 pays : beurre, butter-oil, poudres de lait entier et écrémé destinés à I'alimentation humaine, laits concentrés sucré et non sucré. Tome 2 : l'Afrique noire, I'Afrique australe et le Maghreb. Paris, France, Cfce, 212 p., n.d.

3. FAO, 1981. Agriculture : horizon 2000. Rome, Italie, Fao, 178 p. (Etudes de développement économique et social, $n^{\circ} 23$ )

4. FAO, 1995. Annuaire du commerce 1994. Rome, Italie, Fao, 385 p. (vol. 48)

5. FAO, 1996. Annuaire de la production 1995. Rome, Italie, Fao, 235 p. (vol. 49)

6. FAO, 1996. Le marché mondial de la viande : situations et perspectives 1995. Rome, Italie, Fao, n. p.

7. FAO, 1996. Rapport de la $16^{\mathrm{e}}$ session du Groupe intergouvernemental sur la viande, Bologne, Italie, 8-10 mai 1996. Rome, Italie, Fao, $13 \mathrm{p}$

8. FAO, 1996. Southern African Meat Board. Livestock and meat trade in the Southern African developement community. Rome, Italy, FAO, 60 p. + annexes.

9. FAOSTAT. Statistics database. Sur serveur web de la Fao. Adresse U rl : http://www.fao.org (consulté le 23 septembre 1997).

10. LACROUTS M., 1969. Problème de la commercialisation du bétail en Afrique. Revue Elev. Méd. vét. Pays trop., 22 : 127-144.
11. SARNIGUET J., 1990. Effets des importations de viandes du marché mondial sur les échanges de la région Afrique de l'O uest et centrale 1970/1987. Paris, France, Solagral (Réseau stratégies alimentaires), n.p. (D ocuments du groupe « Elevage »).

12. SO LAGRAL, 1994. Bétail et viandes en Afrique de l'Ouest et du Centre : enjeux et perspectives. Paris, France, Solagral (Réseau stratégies alimentaires), 7 fiches.

13. TACHER G., LETENNEUR L., 1997. Étude sur la compétitivité des filières de productions animales en Afrique subsaharienne et à Madagascar. Phase 1 : rapport de synthèse. Cirad-emvt, Montpellier, France, Bdpa-Scetagri, Paris, France, $142 \mathrm{p}$.

14. TACHER G., LETENNEUR L., 1999. Le secteur des productions animales en Afrique subsaharienne des indépendances à 2020. I. Place de l'Afrique subsaharienne dans les échanges mondiaux et évolution du secteur élevage. Revue Elev. M éd. vét. Pays trop., 52 : 279-290.

Reçu le 15.9.98, accepté le 21.12.99

\section{E R R A T U M}

Dans la partie I de ce projet (Revue Elev. Méd. vét. Pays trop., 1999 52 (3-4) : 279-290), page 288 tableau IX, lire : * en 1000 tonnes équivalent carcasse. 


\section{Summary}

Tacher G., Letenneur L. Livestock sector in sub-Saharan Africa, from the Independences to 2020. II. Exchanges approached by subregional zones

Sub-Saharan Africa was zoned into seven subregions (W est, Center-W est, Center-North, East, Center, South, and Madagascar) in order to specify its great exchange and consumption diversity. Zoning was based on the demand for animal products, the homogeneity and complementariness of exchanges, and political alliances. Imports and exports mainly concern meat with a wide diversity depending on the zone: surplus balance in the Eastern zone, deficit balance in Central Africa. With regard to milk products, all the identified subregions are in deficit. The global deficit amounted to 428 million dollars in 1994. Leathers and hides represent $15 \%$ of the exports, of which $67 \%$ originate from Austral Africa. They represent a positive balance and compensate for the import/export balance of poultry. When examined by subregions, there are several types of consumption and there is a wide variety in the amounts of animal proteins consumed daily per inhabitant: from $4.2 \mathrm{~g}$ in the central zone to $13.8 \mathrm{~g}$ in the IGAD (Intergovernmental Authority on D rought) zone. The consumption evolution shows a high increase in white meat (poultry, pork) consumption in the diets in most zones. Depending on the zone, supply varies greatly with regard to the composition and number of animal species, the products and imports as well. The two large importing subregions are Central and Austral Africa.

Key words: Animal production - Feed consumption - Import Export - Zoning - Animal protein - Supply - Africa south of Sahara.

\section{Resumen}

Tacher G., Letenneur L. El sector de las producciones animales en Africa subsahariana desde las independencias hasta el 2020. II. Enfoque de los intercambios por zonas sub regionales

Para definir la gran diversidad de los intercambios y de los consumos en el interior de Africa subsahariana, se llevó a cabo una categorización de las zonas en siete sub regiones (O este, Centro-O este, Centro-N orte, Este, Centro, Sur, Madagascar), basada en la demanda de productos animales, en la homogeneidad y la complementariedad de los intercambios, así como tomando en consideración las uniones políticas. Las importaciones y exportaciones corresponden en su mayoría a las carnes, con una gran diversidad según las zonas: saldo excedente para la zona oriental, saldo deficitario en Africa central. Todas las sub regiones identificadas fueron deficitarias en productos lácteos, con un déficit global de 428 millones de dólares americanos en 1994. Los cueros y las pieles represen$\tan 15 \%$ de las exportaciones, $67 \%$ de éstas son realizadas en Africa austral. Estas representan un saldo positivo y compensan las importaciones netas de aves. Los consumos examinados por sub región muestran varios tipos de consumo y una gran diversidad en las cantidades de proteínas animales consumidas por día y por habitante, yendo de 4,2 g en la zona central a 13,8 g en la zona de Igad (Autoridad intergubernamental en Sequías). La evolución de los consumos muestra un fuerte aumento del consumo de carnes blancas en los regímenes alimenticios en la mayoría de las zonas. La oferta muestra una gran diversidad entre las siete zonas, tanto en la composición, efectivos de las especies animales y en los productos, como en las importaciones. Las dos grandes sub regiones importadoras son Africa central y Africa austral.

Palabras clave: Producción animal - Consumo de piensos Importación - Exportación - Zonificación - Proteínas de origen animal - O ferta - Africa al sur del Sahara. 